Research Paper

\title{
Application of cAMP-dependent catalytic subunit $\beta$ (PRKACB) Low Expression in Predicting Worse Overall Survival: A Potential Therapeutic Target for Colorectal Carcinoma
}

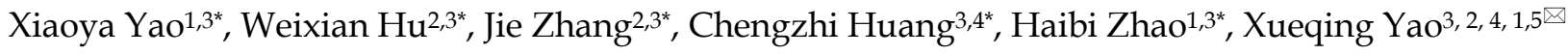 \\ 1. School of Bioscience and Bioengineering, South China University of Technology, Guangzhou, Guangdong, People's Republic of China. \\ 2. The Second School of Clinical Medicine, Southern Medical University, Guangzhou, People's Republic of China. \\ 3. Department of General Surgery, Guangdong Provincial People's Hospital, Guangdong Academy of Medical Sciences, Guangzhou, Guangdong, People's \\ Republic of China. \\ 4. School of Medicine, South China University of Technology, Guangzhou, Guangdong, People's Republic of China. \\ 5. Shantou University Medical College, Shantou, Guangdong, People's Republic of China. \\ *These authors have contributed equally to this work. \\ $\triangle$ Corresponding author: Xueqing Yao, MD, Ph.D., Department of General Surgery, Guangdong Provincial People's Hospital, Guangdong Academy of Medical \\ Sciences, Guangzhou 510080, China; Email: syyaoxueqing@scut.edu.cn. \\ () The author(s). This is an open access article distributed under the terms of the Creative Commons Attribution License (https://creativecommons.org/licenses/by/4.0/). \\ See http://ivyspring.com/terms for full terms and conditions.
}

Received: 2020.03.19; Accepted: 2020.05.25; Published: 2020.06.07

\begin{abstract}
Low expressions of PRKACB are related to the occurrence of various human malignancies. However, the prognostic value of PRKACB expression in colorectal cancer (CRC) patients remains controversial. In this analysis, PRKACB expression in CRC tumors was evaluated across the GEO, TCGA, and Oncomine databases, and a PRKACB survival analysis was performed based on the TCGA profile. We detected PRKACB in 7 GEO series (GSE110225, GSE32323, GSE44076, GSE9348, GSE41328, GSE21510, GSE68468) and TCGA spectra (all $P<0.05$ ). A meta-analysis performed in the Oncomine database revealed that PRKACB was significantly up-regulated in neoplastic tissues compared to normal tissues (all $P$ <0.05). A Kaplan-Meier analysis demonstrated that lower PRKACB expression in tumors was significantly associated with poorer overall survival $(O S)$ in patients with $C R C(P<0.05)$. A subgroup analysis showed that low expression of PRKACB correlated with poor 1-, 3-, and 5-year OS (all P $<0.05$ ). Furthermore, in males $(P=0.0083)$, whites $(P=0.0463)$, and non-mucinous adenocarcinoma patients $(P$ $=0.0108)$, the down-regulation of PRKACB expression was more significant for the OS prognostic value. Conclusion: PRKACB is down-regulated in tumors and associated with worsening OS in CRC patients.
\end{abstract}

Key words: PRKACB; colorectal carcinoma; survival; therapeutic target

\section{Introduction}

Colorectal cancer (CRC) is amongst the most prevalent digestive tract malignancies and it is the world's second most deadly cancer with almost 900000 deaths recorded annually [1]. Despite formidable advances in imaging techniques, surgery and multimodal therapy, the overall survival for patients with advanced CRC remains low. A study showed that the incidence of CRC in China has constantly been on the rise over the past three decades and equally predicted a further increase in the near future [2]. According to the 2015 cancer statistics [3], it is estimated that about 2,376,300 new CRC cases and 191,000 CRC related deaths occurred in China, accounting for nearlyone-tenth of the global CRC burden. Even though outstanding radiological, surgical and multimodal therapeutic advances have recently been mad, the overall survival rates for patients with late stage CRC remain substantially low at $\sim 8-9 \%$ [4]. A precise estimation of the prognosis plays an important role in the diagnostic and therapeutic management of CRC patients. Thus, identifying reliable and practical prognostic 
biomarkers and revealing novel CRC treatment targets is urgently required $[5,6]$.

The protein kinase cAMP-dependent catalytic subunit $\beta$ (PRKACB) encoding the CAMP-dependent protein kinase catalytic subunit $\beta$ (PRKACB) is a member of the serine/threonine protein kinase family [7]. It serves as a key regulatory point and is involved in cell proliferation, differentiation, regulation of apoptosis, and is closely related to physiological and pathological processes such as cell growth, gene expression, tumor proliferation and metastasis [8]. Recently, the presence of the PRKACA and PRKACB fusion genes has been detected in various cancers such as bile duct cancers, fibrolamellar hepatocellular carcinoma, and pancreatic cancers, and there is a possibility that this might be implicated in the pathogenesis of cancer [9-11]. Some researches declared that PRKACB mutations could cause adrenal and bile duct tumors [12,13], and that high expressions of PRKACB is related to drug resistance in patients diagnosed with breast cancer [14]. Previous studies have confirmed that the c-MYC was directly activated by PRKACB, which then induced neoplasia [15,16]. Conversely, another study suggested that the PRKACB might act as a tumor suppressor gene in non-small cell lung cancers [17]. Nonetheless, the exact role played by PRKACB in colorectal cancer is still unknown. Thus, to evaluating the prognostic value of the PRKACB in CRC patients is fundamental.

To help elucidate the possible relationship between the PRKACB expression and CRC patient outcomes, we identified the PRKACB expression in GEO, Oncomine and TCGA databases and performed a survival analysis based on the TCGA profile, with hopes of providing useful insights into the development of colorectal cancer.

\section{Materials and Methods}

\section{Data resource and Description}

GEO microarray series (GSE110225, GSE32323, GSE44076, GSE9348, GSE41328, GSE21510, GSE68468) containing CRC tumor and non-tumor samples were obtained from the National Center for Biotechnology Information's (NCBI) Gene Expression Omnibus
(GEO, https://www.ncbi.nlm.nih.gov/geo/). Platforms and samples of GEO series were summarized in Table 1.

All of the publicly available colorectal cancer RNA-Seq data information were downloaded from The Cancer Genome Atlas (TCGA) official website (https://cancergenome.nih.gov/) before December 17, 2019, through the GDC Data Transfer Tool [18]. TCGA RNAseq data was comprised of 568 tumor samples and 44 non-tumor samples.

\section{Bioinformatics analysis for identifying PRKACB expression}

Raw CEL files of the microarray from each GEO dataset were normalized by the quantile method of Robust Multichip Analysis (RMA) from the R affy package [19] and the normalized gene expression levels were presented as log2-transformed values by RMA. Gene expression of PRKACB was determined by tumor and non-tumor samples comparison from the R Limma package [20].

The $R$ language version 3.6.1 edgeR package $[21,22]$ was used to compare the mRNA expression of tumor and non-tumor samples from TCGA.

Studies comparing PRKACB between tumor and non-tumor samples in colorectal cancer were selected with a threshold of $\mathrm{p}$-Value $\leq 1 \mathrm{E}-4$, fold change $\geq 2$ and top $10 \%$ gene rank in the Oncomine database (https://www.oncomine.org/).

\section{Survival analysis}

The clinical data and PRKACB RNA Seq V2 data of 382 colorectal cancer patients (TCGA, Provisional) were downloaded from the cBioPortal database $[23,24]$ (http://www.cBioPortal.org/), and the patients' information were matched with the sample ID by VLOOKUP index in EXCEL. After deleting some missing data, the PRKACB expression values were ranked from top to bottom and the median was taken as the cutoff point, these patients were then classified into a low expression group and high expression group to analyze the correlation between PRKACB expression with survival rates and clinical pathological characteristics.

Table 1. Details of GEO series included in this analysis

\begin{tabular}{lllll}
\hline GEO series & Contributor(s), Year & Tumor & Non-tumor & Platform \\
\hline GSE110225 & Vlachavas E, 2018 & 30 & 30 & [HG-U133A] Affymetrix Human Genome U133A Array \\
& & & & [HG-U133_Plus_2] Affymetrix Human Genome U133 Plus 2.0 Array \\
GSE32323 & Ahmed K et al., 2012 & 17 & 17 & [HG-U133_Plus_2] Affymetrix Human Genome U133 Plus 2.0 Array \\
GSE44076 & Solé X et al., 2014 & 98 & 98 & [HG-U219] Affymetrix Human Genome U219 Array \\
GSE9348 & Hong Y et al., 2010 & 70 & 12 & [HG-U133_Plus_2] Affymetrix Human Genome U133 Plus 2.0 Array \\
GSE41328 & Lin G et al., 2012 & 5 & 5 & [HG-U133_Plus_2] Affymetrix Human Genome U133 Plus 2.0 Array \\
GSE21510 & Tsukamoto S, 2011 & 19 & 25 & [HG-U133_Plus_2] Affymetrix Human Genome U133 Plus 2.0 Array \\
GSE68468 & NA, 2015 & 44 & 44 & [HG-U133A] Affymetrix Human Genome U133A Array \\
\hline
\end{tabular}




\section{Identification of PRKACB-related genes}

RNA-Seq data information of 568 tumor samples and 44 non-tumor samples were downloaded from TCGA official website and the edgeR package [21,22] was used to compare the mRNA expression of the tumor and non-tumor samples to screen for differential expression genes (DEGs). The P-value $<0.05$ and $|\log \mathrm{FC}|>1$ were chosen as cut-off criteria. The Spearman coefficients of the DEGs and PRKACB were calculated, whilst DEGs with $p$-value $<0.05$ were defined as PRKACB-related genes.

\section{KEGG/GO biological process enrichment}

The Database for Annotation, Visualization, and Integrated Discovery (DAVID, http://david.ncifcrf. gov) (version 6.8) [25] is an online functional annotation tool that we applied for Gene Ontology (GO) enrichment analysis, including biological process (BP), cellular components (CC), and molecular function (MF). The DAVID database was also used to a perform pathway enrichment analysis. $P$-value $<0.05$ was considered as the threshold.

\section{Protein-protein interaction (PPI) network construction}

STRING (http://string-db.org) (version 11.0) [26] is an online biological database for the prediction of known and unknown protein interaction relationships. The PRKACB-related genes were uploaded to the STRING website to analyze the interactions between those proteins. The minimum required interaction score was set to 0.400 (medium confidence) and the protein nodes undergoing no interaction with other proteins were removed. Next, the PPI pairs were inputted into the Cytoscape software (http://www.cytoscape.org) (version 3.7.1) [27] to construct a PPI network and the top $10 \mathrm{hub}$ genes were identified in accordance with the Cytoscape plug-in (degrees ranking of cytoHubba).

\section{Gene Co-expression Network Analysis}

The mRNA expression of 7 complete samples from the Colorectal Adenocarcinoma (TCGA, Provisional) database was used to conduct an analysis by the Co-expression online analysis function in the cBioPort database (http:// www.cBioPortal.org/) [24]. $P$ value $<0.05$ was considered as the threshold. Genes with Spearman correlation coefficients and PRKACB expression greater than 0.9 were screened and uploaded to Cytoscape software (http://www. cytoscape.org) (version 3.7.1) [27] to draw a gene co-expression network.

\section{Results}

\section{PRKACB expression comparison}

The details of the GEO series included in this analysis were summarized in Table 1 . As illustrated in Figure 1, the expression of PRKACB in tumor samples was all significantly lower than non-tumor samples in GSE110225, GSE32323, GSE44076, GSE9348, GSE4128, GSE21510, GSE68468 and TCGA datasets (all P < 0.01, Figure 1).
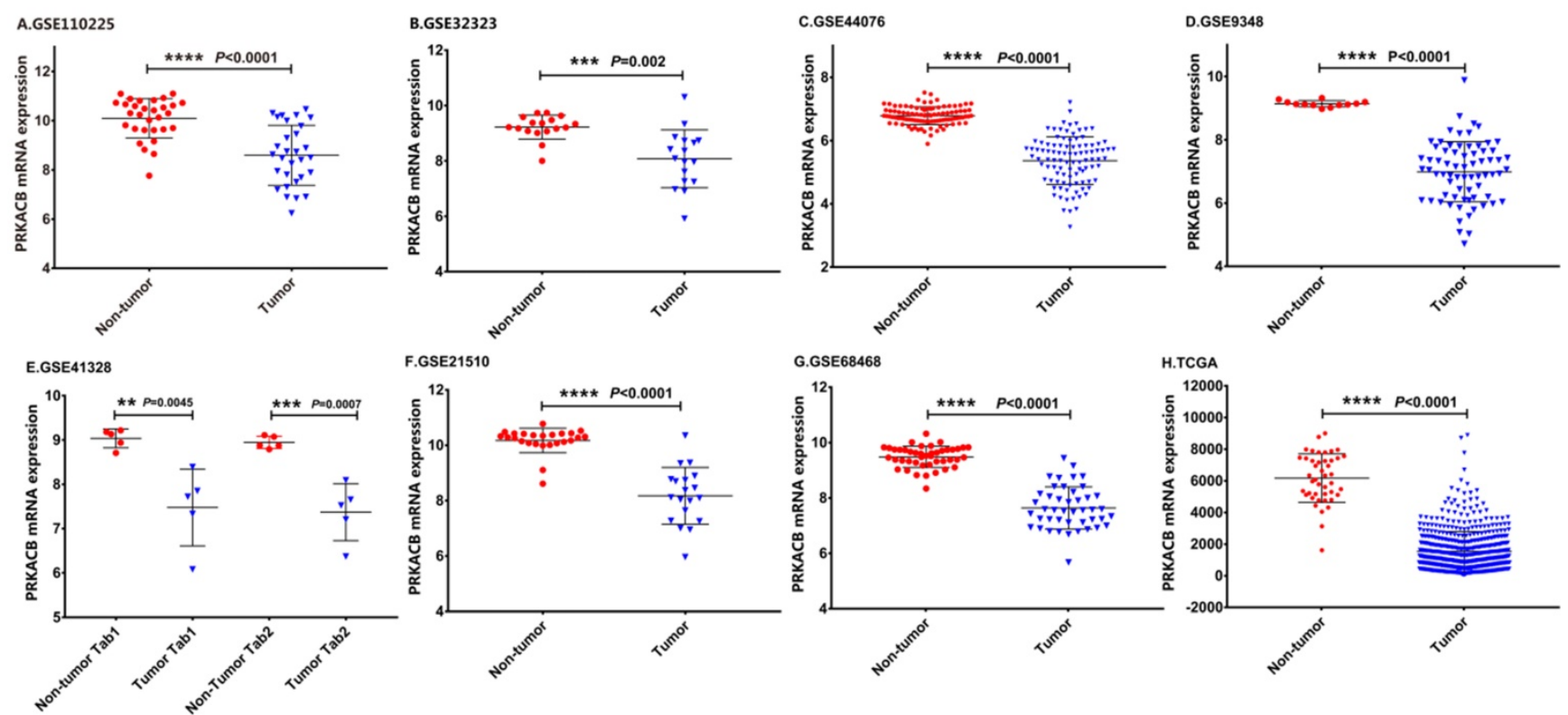

Figure 1. mRNA expression levels of PRKACB between tumor and non-tumor samples in CRC patients on the GEO database series including GSE1 10225 (A), GSE32323 (B), GSE44076 (C), GSE9348 (D), GSE4128 (E), GSE21510 (F), GSE68468 (G), TCGA database (H). 
For validation, we performed a meta-analysis of PRKACB expression in 15 analyses with a threshold set as p-Value $\leq 1 \mathrm{E}-4$, fold change $\geq 2$ and top $10 \%$ gene rank in the Oncomine database. As displayed in Figure 2 and compared with its value in normal tissues, PRKACB was significantly downregulated in rectal adenocarcinoma $(\mathrm{P}<0.0001$, Figure $2 \mathrm{~B})$, rectal carcinoma $(\mathrm{P}<0.0001$, Figure $2 \mathrm{G})$, colon carcinoma $(\mathrm{P}<0.0001$, Figure 2E-G), colon adenoma $(\mathrm{P}<0.0001$, Figure 2E), colorectal carcinoma $(\mathrm{P}<0.0001$, Figure $2 \mathrm{C}-\mathrm{D})$, and colorectal adenocarcinoma $(\mathrm{P}<0.0001$, Figure 2D). Notwithstanding, the difference of PRKACB expression in rectal carcinoma has no value $(\mathrm{P}=0.2021$, Figure $2 \mathrm{~F})$. Besides, the PRKACB was also downregulated in cecal carcinoma $(\mathrm{P}<0.0005$, Figure $2 \mathrm{~F})$ and cecal adenocarcinoma $(\mathrm{P}<0.0001$, Figure $2 \mathrm{G})$.

\section{Associations between PRKACB and survival in CRC patients}

The results outlined that low expression of PRKACB in tumor tissues was considerably associated with poor disease-free survival (log rank $P$ $=0.0025$, Figure $3 \mathrm{~A})$ and overall survival $(\log \operatorname{rank} \mathrm{P}=$ 0.0186 , Figure $3 \mathrm{~B}$ ) in patients with CRC. Moreover, a subgroup analysis revealed that the downregulation of PRKACB in tumor tissue was a risk factor for reduced 1 year $(\log \operatorname{rank} \mathrm{P}=0.0100, \mathrm{HR}=9.273$ (2.684-32.04), Figure 3C), 3 years (log rank $P=0.0041$, $\mathrm{HR}=3.477$ (1.635-7.393), Figure 3D), and 5 years (log rank $\mathrm{P}=0.0083, \mathrm{HR}=2.677$ (1.367-5.244), Figure $3 \mathrm{C})$ OS in CRC patients.

By the same token, we performed subgroup survival analyses in different populations. As shown in Figure 4, downregulation of PRKACB was associated with poorer survival in males and white patients, while no significant differences were found in females and black patients. Moreover, low PRKACB levels significantly contributed to worse OS in CRC patients without non-mucinous cancers $(\mathrm{HR}=2.557(1.328-4.924), \log$-rank $\mathrm{P}=0.0108$, Figure 4E) and the down-regulation of PRKACB was observed in patients with stage III-IV colorectal cancer $(\mathrm{HR}=2.931$ (1.357-6.333), log-rank $P=0.0145$, Figure $4 G$ ), but not identified as risk factors for patients with stage I-II colorectal cancer $(\mathrm{P}>0.05$, Figure $4 \mathrm{H})$.

\section{Association between PRKACB and clinicopathological features in CRC patients}

As delineated in Table 2, there are more male cases in the PRKACB low group $(64.85 \%$ vs. $46.99 \%, \mathrm{P}$ $=0.001)$ and PRKACB low group patients were significantly older than those in the PRKACB high group (66yr vs. 61.5yr, $\mathrm{P}=0.022$ ). However, $\mathrm{BMI}$, tumor stage, lymph node stage, metastasis stage, AJCC stage, lymphovascular invasion, perineural invasion, vascular invasion, race category, person neoplasm status were not significantly different in PRKACB expression ( $\mathrm{P}>0.05)$.

\section{KEGG/GO biological process enrichment}

The KEGG pathway enrichment of PRKACB interactive genes showed that neuroactive ligandreceptor interactions, pancreatic secretions, bile secretions, mineral absorption, salivary secretions, cAMP signaling pathways, glutamatergic synapse, GABAergic synapses, retrograde endocannabinoid signaling, circadian entrainment, etc. were the most enriched pathways (Figure 5A). Additionally, GO analysis results proved that PRKACB-related genes were significantly enriched in the complement activation classical pathway, proteolysis, complement activation, etc. at BP levels; extracellular region, extracellular space, plasma membrane, etc. at CC levels and antigen-binding, serine-type endopeptidase activity and hormone activity at MF levels (Figure 5B-D).

\section{Protein-protein interaction (PPI) network construction}

A total of 127 genes were filtered into the target genes PPI network complex, containing 100 nodes and 654 edges, 10 hub Genes (PRKACB, ATP2B2, MAPT, PHLPP2, ABCCB, GRIN2A, MYLK, GRIA1, $\mathrm{BCHE}, \mathrm{ADCY} 2$ ) were screened according to Cytoscape 3.7.1 and its plug-in (Ranking degree of cytoHubba).

\section{Gene Co-expression Network Analysis}

Identification of PRKACB co-expressed genes was completed by the cBioPortal database's online analysis function. A total of 182 genes with Spearman correlation coefficients greater than 0.9 (highly correlated) were selected and visualized via Cytoscape (version 3.7.1) (Figure 7). We found that FAM167A, NRIP3, RASL11B, ST13P4, TMEM99 completely correlated with PRKACB (Spearman's Correlation =1) whereas ALPP, C3ORF70, JUND, and ZBTB7A were completely negative correlated with PRKACB (Spearman's Correlation $=-1$ ).

\section{Discussion}

Over these past years, more and more data indicate that PRKACB is involved in the cancerization process of malignant tumors in different systems. PRKACB mutations and gene fusion of PRKACB can lead to adrenal, bile duct, liver, and pancreatic cancers $[12,13]$, meanwhile down-regulation of PRKACB expression may be associated with poor survival in patients with non-small cell lung cancer. Makondi et al. found that targeting PRKACB may increase the 
responsiveness of colorectal tumors to irinotecan treatment [28], and Feng et al. also found pathological expressions of PRKACB in human colorectal cancer tissues infected with nucleobacteria [29]. Consistent with previous studies [30], our study showed that PRKACB had a significant discrepancy of expression in normal and tumor tissues of colorectal cancer, and we also depicted that down-regulation of PRKACB expression is a risk factor for declining 1,3, and 5-year survival rates in patients with CRC. This was equally associated with poor patient-free survival and overall survival. Consequently, we can confirm that PRKACB plays an important role in patients with colorectal cancer and affects the prognosis of patients.

Gene co-expression network analysis established that PRKACB had a completely positive correlation with FAM167A, NRIP3, RASL11B, ST13P4, TMEM99, and a completely negative correlation with ALPP, C3ORF70, JUND, and ZBTB7A. It has been confirmed by studies that NRIP3 and RASL11B play a role in suppressing cancer proliferation in breast cancer and renal cell carcinoma [31,32]. Meanwhile ALPP, JUND, ZBTB7A in gastric cancer, prostate cancer, breast cancer [33-35] and other cancers promote the progress of cancer. Thence, we can boldly speculate that PRKACB plays a synergistic role with these tumor suppressors in inhibiting tumor growth. On the contrary, oncogenes antagonize the tumorsuppressive effect of PRKACB.

Many studies have confirmed that microRNAs can regulate the expression of PRKACB [29,36-38], affect cell proliferation, adhesion, and metastasis, and eventually bring about the development of tumors. Also, studies have found that antisense oligodeoxynucleotides targeted to protein kinase subunits induce growth arrest, apoptosis, and differentiation in a variety of cancer cell lines both in vitro and in vivo [39]. Similarly, we were able to regulate the development of colon cancer by targeting PRKACB, providing an important theoretical basis for the development of colon cancer chemotherapeutic drugs and new anti-EMT therapies.

Table 2. Characteristics of CRC patients between PRKACB high and low groups

\begin{tabular}{|c|c|c|c|c|}
\hline & & High groups $(n=166)$ & Low groups $(n=165)$ & $P$ \\
\hline \multirow[t]{2}{*}{ Gender* } & Male $(n=185)$ & 78 & 107 & 0.001 \\
\hline & Female $(n=146)$ & 88 & 58 & \\
\hline \multirow[t]{3}{*}{ Age, median(IQR), years* } & & $61.5(21.75)$ & $66(17)$ & 0.022 \\
\hline & $\leq 60(\mathrm{n}=139)$ & 80 & 59 & \\
\hline & $>60(\mathrm{n}=192)$ & 86 & 106 & \\
\hline \multirow[t]{4}{*}{$\mathrm{BMI}, \mathrm{kg} / \mathrm{m} 2$} & $<18.5(\mathrm{n}=4)$ & 1 & 3 & 0.836 \\
\hline & $18.5-24.99(\mathrm{n}=86)$ & 44 & 42 & \\
\hline & $25-29.99(n=104)$ & 53 & 51 & \\
\hline & $>29.99(n=60)$ & 29 & 31 & \\
\hline \multirow[t]{5}{*}{ Tumor Stage } & $\mathrm{T} 1(\mathrm{n}=10)$ & 4 & 6 & 0.849 \\
\hline & $\mathrm{T} 2(\mathrm{n}=49)$ & 24 & 25 & \\
\hline & T3 $(n=231)$ & 115 & 116 & \\
\hline & $\mathrm{T} 4(\mathrm{n}=40)$ & 22 & 18 & \\
\hline & Tis $(n=1)$ & 1 & 0 & \\
\hline \multirow[t]{4}{*}{ Lymph Node Stage } & N0 (n=188) & 91 & 97 & 0.513 \\
\hline & $\mathrm{N} 1(\mathrm{n}=87)$ & 46 & 41 & \\
\hline & $\mathrm{N} 2(\mathrm{n}=54)$ & 29 & 25 & \\
\hline & $N X(n=2)$ & 0 & 2 & \\
\hline \multirow[t]{3}{*}{ Metastasis Stage } & M0 (n=228) & 111 & 117 & 0.143 \\
\hline & M1 $(n=40)$ & 26 & 14 & \\
\hline & $M X(n=59)$ & 28 & 31 & \\
\hline \multirow{4}{*}{ AJCC stage } & $I(n=52)$ & 25 & 27 & 0.213 \\
\hline & II $(n=125)$ & 61 & 64 & \\
\hline & III (n=99) & 47 & 52 & \\
\hline & IV $(n=41)$ & 27 & 14 & \\
\hline \multirow[t]{2}{*}{ Lymphovascular invasion } & YES (n=89) & 43 & 46 & 0.910 \\
\hline & $\mathrm{NO}(\mathrm{n}=206)$ & 101 & 105 & \\
\hline \multirow[t]{2}{*}{ Perineural Invasion } & YES $(n=51)$ & 30 & 21 & 0.196 \\
\hline & $\mathrm{NO}(\mathrm{n}=153)$ & 74 & 79 & \\
\hline \multirow[t]{2}{*}{ Vascular invasion } & YES $(n=61)$ & 31 & 30 & 0.674 \\
\hline & $\mathrm{NO}(\mathrm{n}=226)$ & 108 & 118 & \\
\hline \multirow[t]{4}{*}{ Race Category } & WHITE $(n=232)$ & 112 & 120 & 0.479 \\
\hline & BLACK OR AFRICAN AMERICAN (n=56) & 28 & 28 & \\
\hline & ASIAN (n=12) & 8 & 4 & \\
\hline & AMERICAN INDIAN OR ALASKA NATIVE $(n=1)$ & 1 & 0 & \\
\hline \multirow[t]{2}{*}{ Person Neoplasm Status } & TUMOR FREE $(\mathrm{n}=221)$ & 115 & 106 & 0.845 \\
\hline & WITH TUMOR $(\mathrm{n}=71)$ & 36 & 35 & \\
\hline
\end{tabular}




\section{A. Meta-analysis of PRKACB in 15 Analyses}

P-Value : 1E-4

- Fold change : 2

- Gene rank : Top $10 \%$

\begin{tabular}{|c|c|c|c|c|c|c|c|c|c|c|c|c|c|c|c|c|c|}
\hline Median Rank & p-Value & Gene & & & & & & & & & & & & & & & \\
\hline \multirow[t]{2}{*}{387.0} & $3.33 E-28$ & PRKACB & & & & & & & & & & & & & & & \\
\hline & & & 1 & 2 & 3 & 4 & 5 & 6 & 7 & 8 & 9 & 10 & 11 & 12 & 13 & 14 & 15 \\
\hline
\end{tabular}

Legend

1. Rectal Adenocarcinoma vs. Normal Gaedcke Colorectal, Genes Chromosomes Cancer, 2010

2. Colorectal Carcinoma vs. Normal Hong Colorectal, Clin Exp Metastasis, 2010

3. Cecum Adenocarcinoma vs. Normal Kaiser Colon, Genome Biol, 2007

4. Colon Adenocarcinoma vs. Normal Kaiser Colon, Genome Biol, 2007

5. Colon Mucinous Adenocarcinoma vs. Normal Kaiser Colon, Genome Biol, 2007

6. Rectosigmoid Adenocarcinoma vs. Normal Kaiser Colon, Genome Biol, 2007

7. Colorectal Carcinoma vs. Normal Skrzypczak Colorectal, PLoS One, 2010

8. Colon Adenoma Epithelia vs. Normal Skrzypczak Colorectal 2, PLoS One, 2010

9. Colon Adenoma vs. Normal Skrzypczak Colorectal 2, PLoS One, 2010

10. Colon Carcinoma Epithelia vs. Normal Skrzypczak Colorectal 2, PLoS One, 2010

11. Colon Carcinoma vs. Normal Skrzypczak Colorectal 2, PLoS One, 2010

12. Cecum Adenocarcinoma vs. Normal TCGA Colorectal, No Associated Paper, 2011

13. Colon Adenocarcinoma vs. Normal TCGA Colorectal, No Associated Paper, 2011

14. Colon Mucinous Adenocarcinoma vs. Normal TCGA Colorectal, No Associated Paper, 2011

15. Rectal Adenocarcinoma vs. Normal TCGA Colorectal, No Associated Paper, 2011

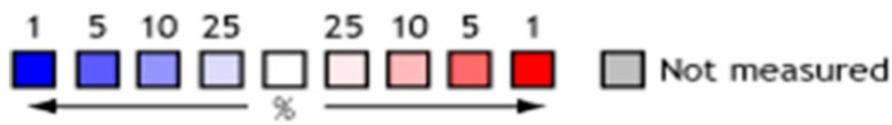

The rank for a gene is the median rank for that gene across each of the analyses.

The p-Value for a gene is its p-Value for the median-ranked analysis.

B. Gaedcke Colorectal

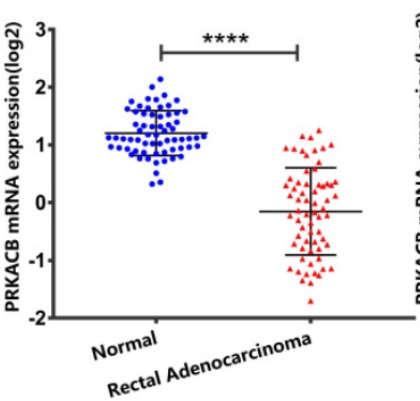

E. Skrzypczak Colorectal 2

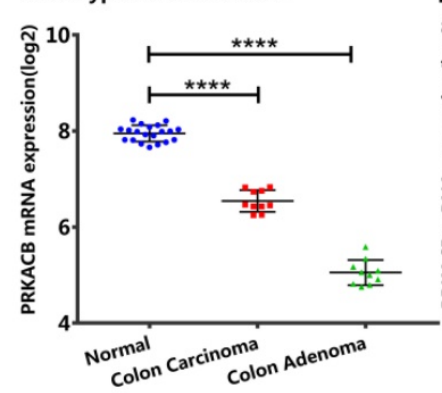

C. Hong Colorectal

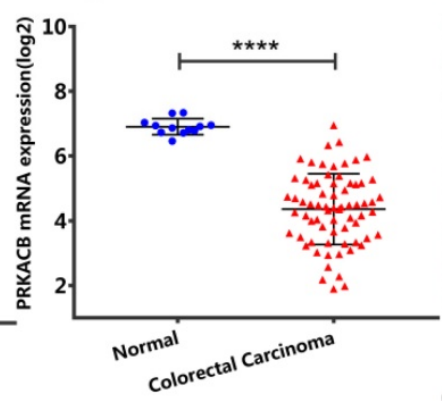

D. Skrzypczak Colorectal

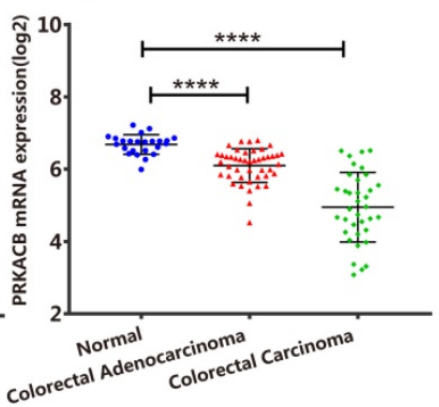

G. TCGA Colorectal

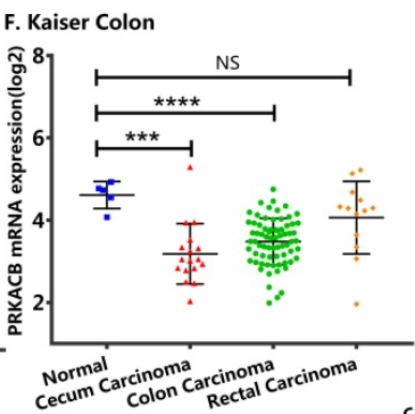

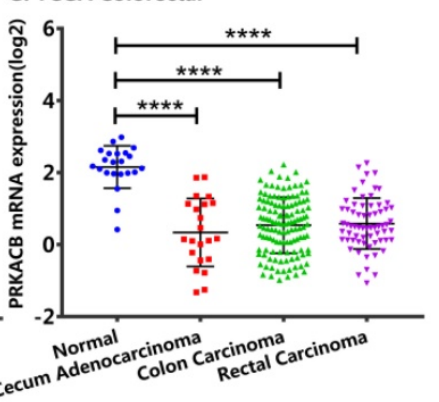

Figure 2. Comparison of PRKACB mRNA expression levels across 15 analyses in the Oncomine database. Meta-analysis of PRKACB expression in 15 analyses (A); PRKACB levels in Gaedcke Colorectal (B), Hong Colorectal (C), Skrzypczak Colorectal (D), Skrzypczak Colorectal 2 (E), Gaedcke Colorectal (F) and, TCGA Colorectal (G). Note: In the Kaiser Colon study, Colon Adenocarcinoma $(n=41)$, Colon Mucinous Adenocarcinoma $(n=13)$, Colon Signet Ring Cell Adenocarcinoma ( $n=2)$, Colon Small Cell Carcinoma $(n=2)$, Rectosigmoid Adenocarcinoma $(n=10)$, Rectosigmoid Mucinous Adenocarcinoma $(n=2)$ were all merged into Colon Carcinoma; Rectal Adenocarcinoma ( $n=8)$, Rectal Mucinous Adenocarcinoma $(n=4)$, Rectal Signet Ring Cell Adenocarcinoma $(n=1)$ were merged into Rectal Carcinoma; In the TCGA Colorectal study, Colon Adenocarcinoma $(n=101)$, Colon Mucinous Adenocarcinoma $(n=22)$, Rectosigmoid Adenocarcinoma $(n=3)$, Rectosigmoid Mucinous Adenocarcinoma ( $n=1)$ were merged into Colon Carcinoma; Rectal Adenocarcinoma $(n=60)$, Rectal Mucinous Adenocarcinoma $(n=6)$ were equally merged into Rectal Carcinoma. 

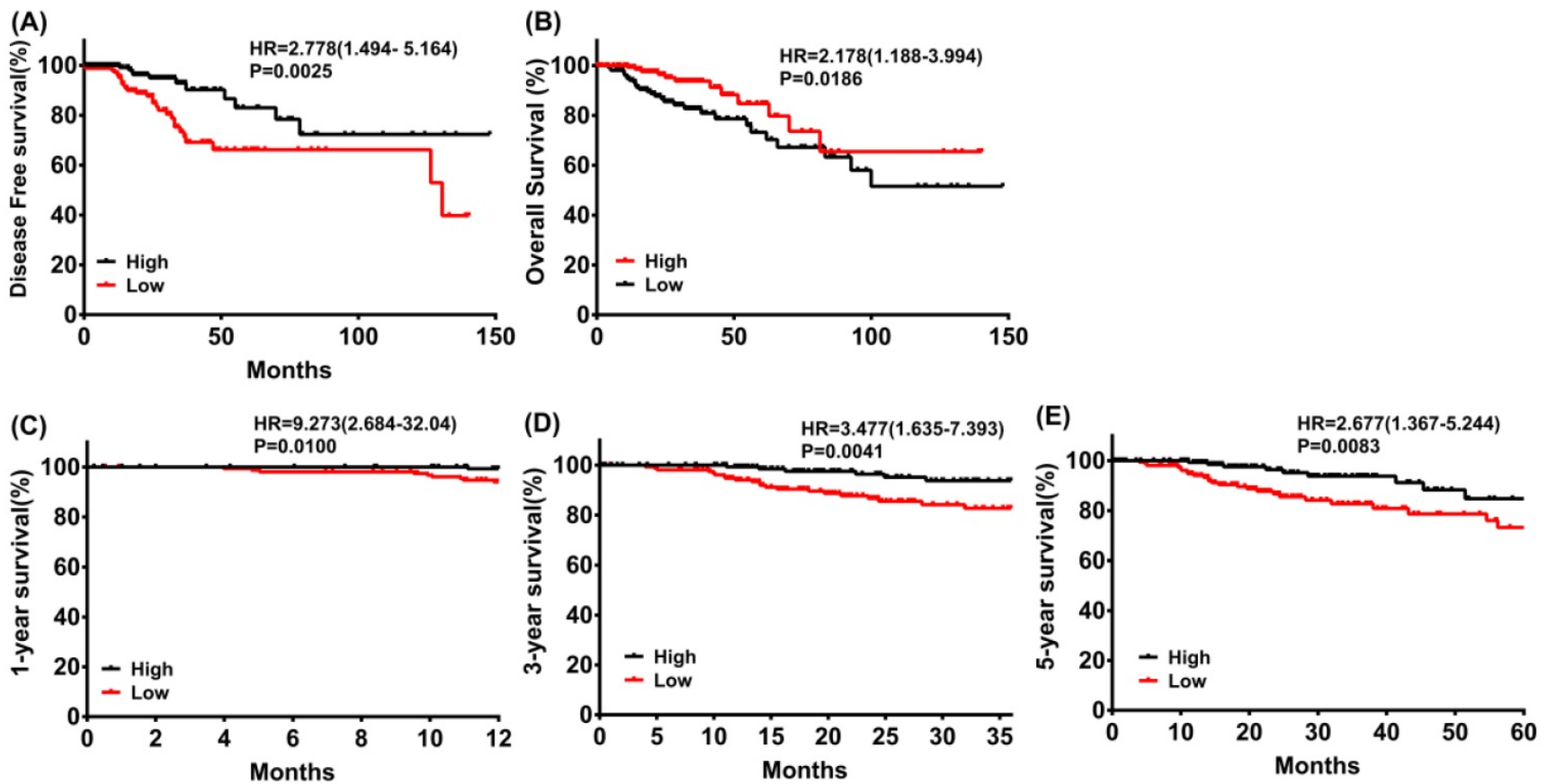

Figure 3. Disease-free survival (A) and overall survival (B) of CRC patients grouped by PRKACB median cutoff in TCGA database; 1-year (C), 3-year (D) and 5-year (E) overall survivals comparison between high and low PRKACB groups.
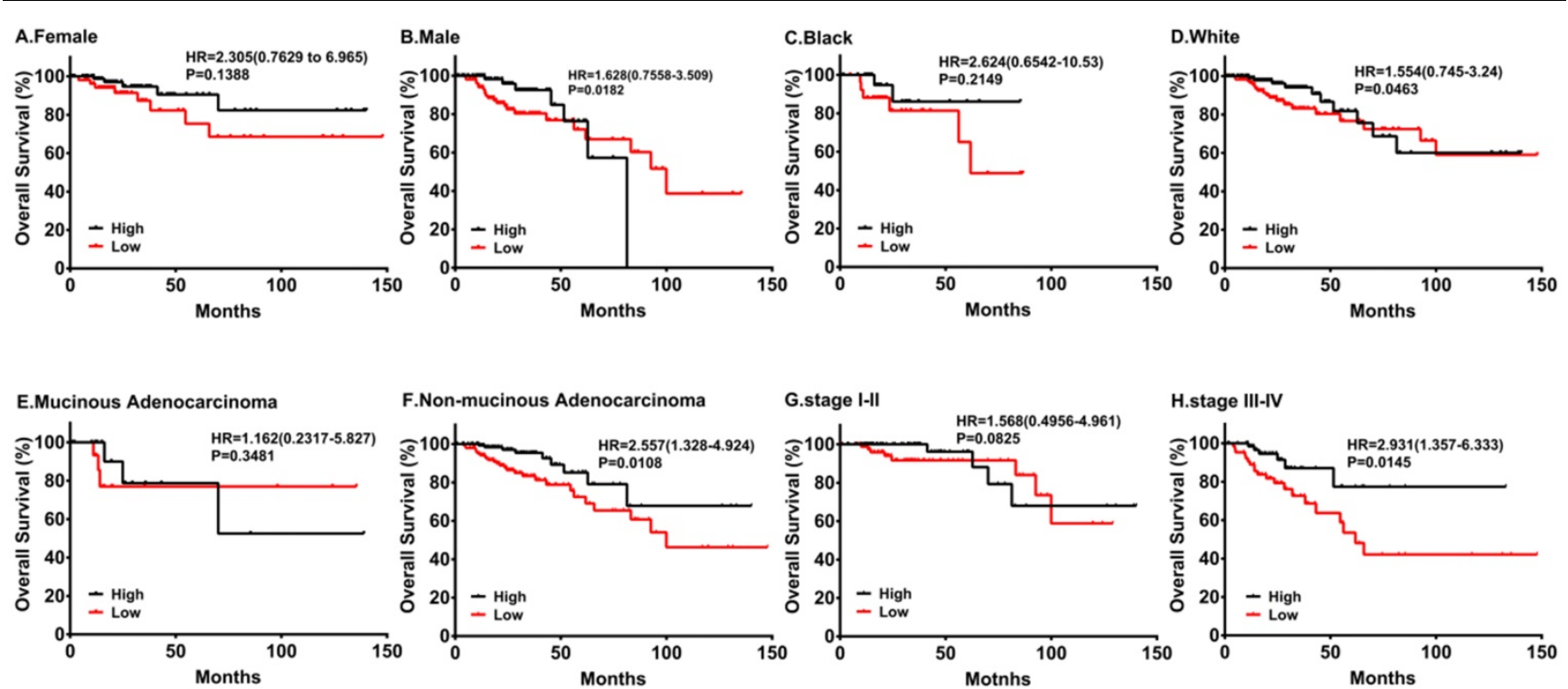

Figure 4. Subgroup analyses of overall survival comparison in different populations [gender (A, B), race (C, D) and cancer type (E, F)] and different stage (G, $H)$ with PRKACB median cutoffs in CRC patients.

The protein kinase cAMP-dependent catalytic subunit $\beta$ (PRKACB) encoding the cAMP-dependent protein kinase catalytic subunit $\beta$ (PRKACB) is a member of the serine/threonine protein kinase family [7]. Activation of PKA catalytic activity is initiated by any signal causing an increase in intracellular cAMP concentration. The traditional view of PKA activation is that two cAMP molecules bind to each R subunit, causing a conformational change in the $\mathrm{R}$ subunit dimer, and the $C$ subunits are released and become catalytically active through exposure of their active sites [40]. PKs catalyze the transfer of phosphate groups onto Ser, Thr, or Tyr residues of target proteins. Phosphorylation of substrates represents a key regulatory mechanism in all eukaryotic cells ${ }^{41}$ and the various PKs target different substrates with a multitude of biological effects. In addition, studies have shown that PKA-mediated tamoxifen resistance in breast cancer is caused by 305 serine (S305) phosphorylation of Era [42]. Similarly, phosphorylation of PRKACB has also been found in neurological diseases [37]. From a physiologic aspect, PRKACB may mainly regulate protein phosphorylation/dephosphorylation through the 
cAMP pathway. Unfortunately, we are yet to conduct experimental studies exploring the potential carcinogenic mechanism of PRKACB in the development of liver cancer. Even after taking into account previous reports, we are pragmatic on suggesting the hypothesis that low expression of PRKACB leads to poorer prognoses in CRC patients.
(A)

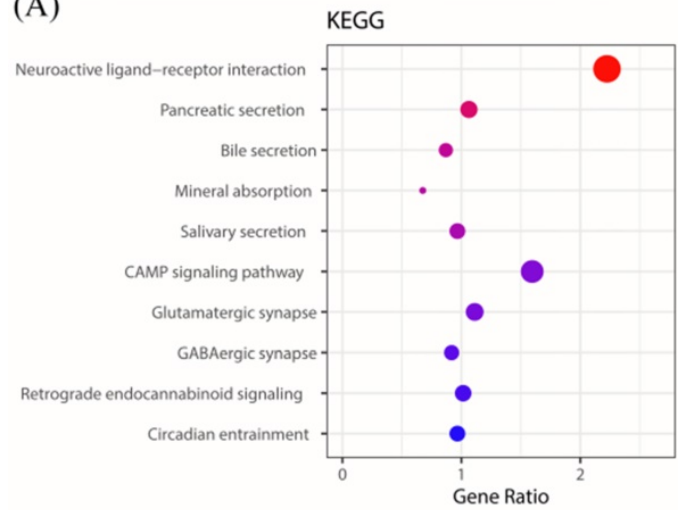

(C)

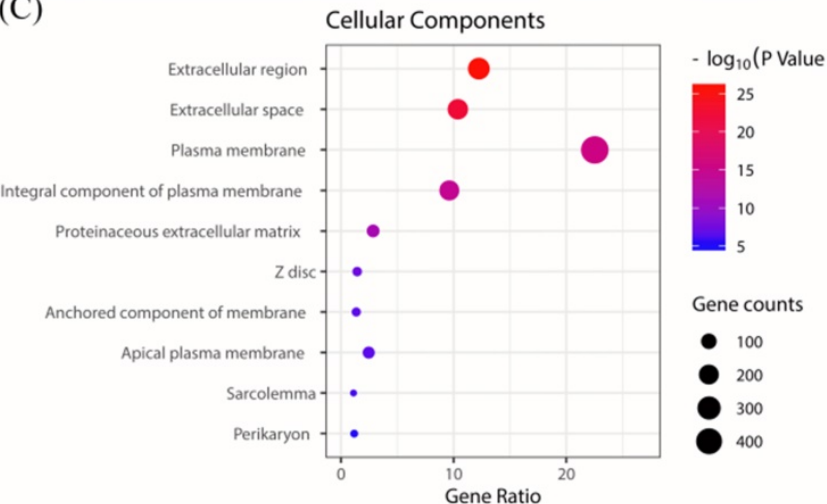

(B)

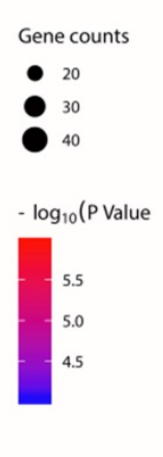

(D)

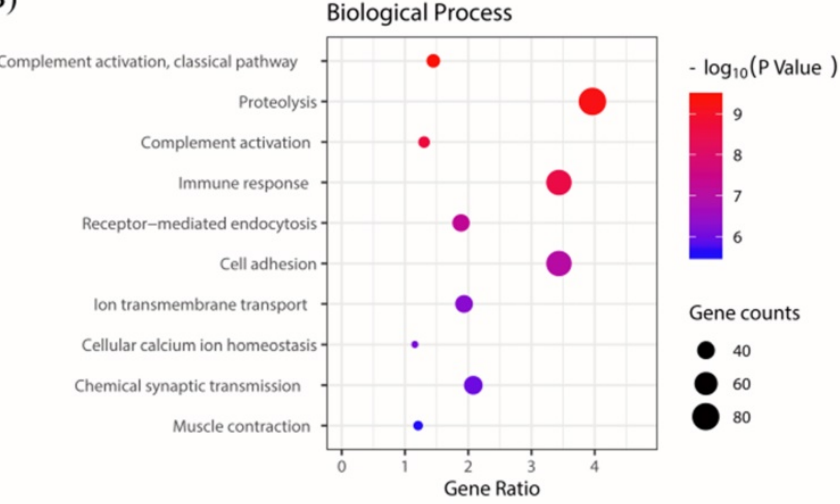

Molecular Function

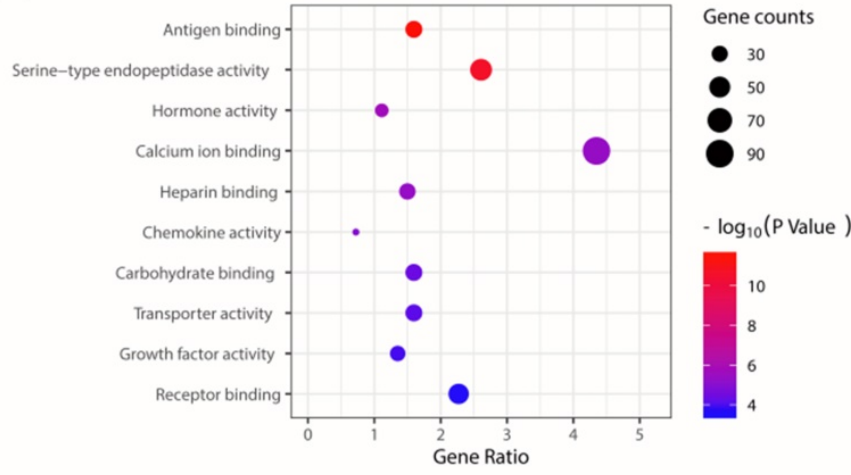

Figure 5. KEGG and GO biological function enrichment analyses of PRKACB related genes. KEGG signal pathway enrichment analysis (A); Biological process enrichment analysis (B), Cell component enrichment analysis (C) and molecular function enrichment analysis (D).

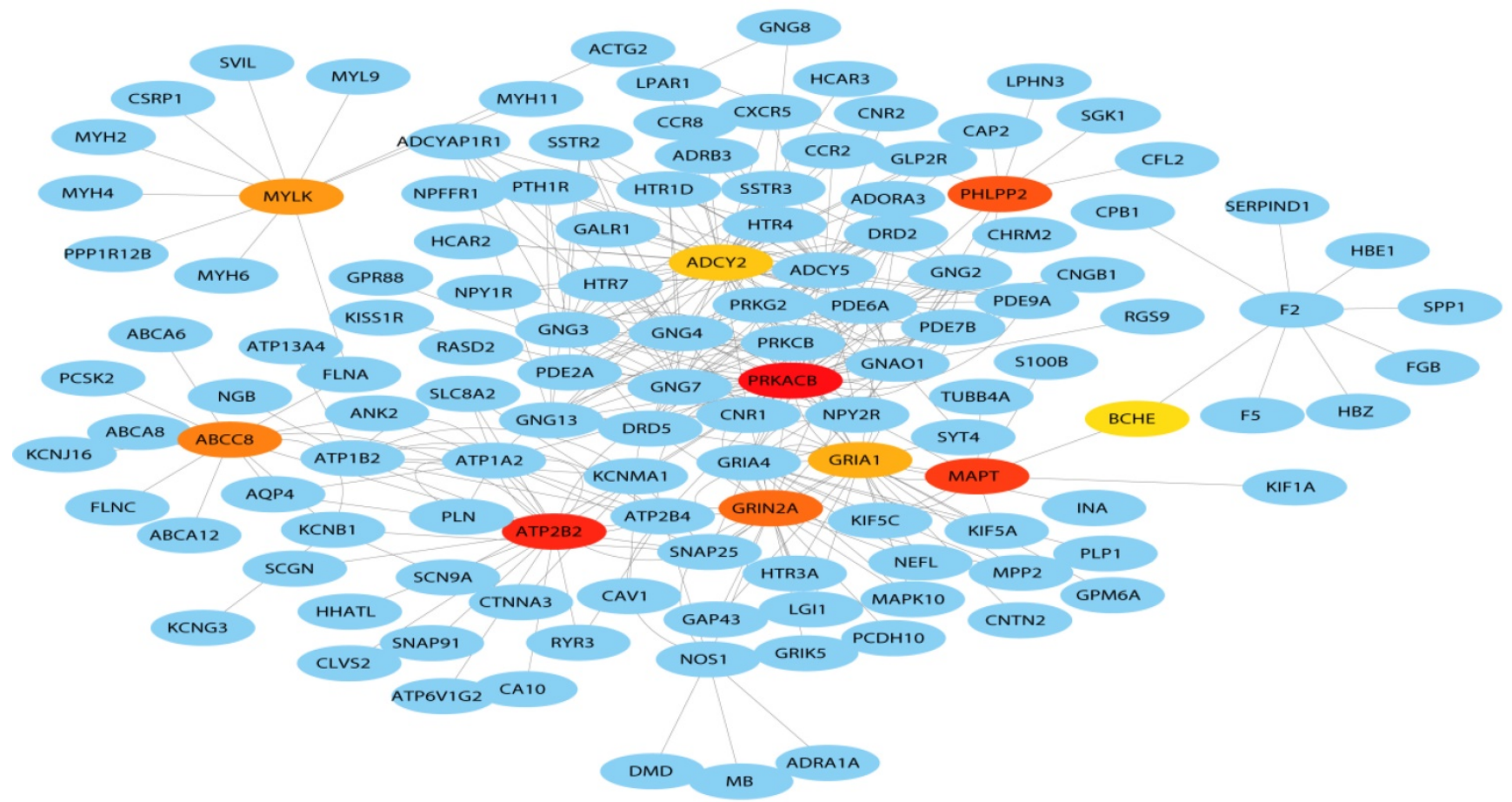

Figure 6. Hub genes of the PPI network. The darker the color, the bigger the degrees. 


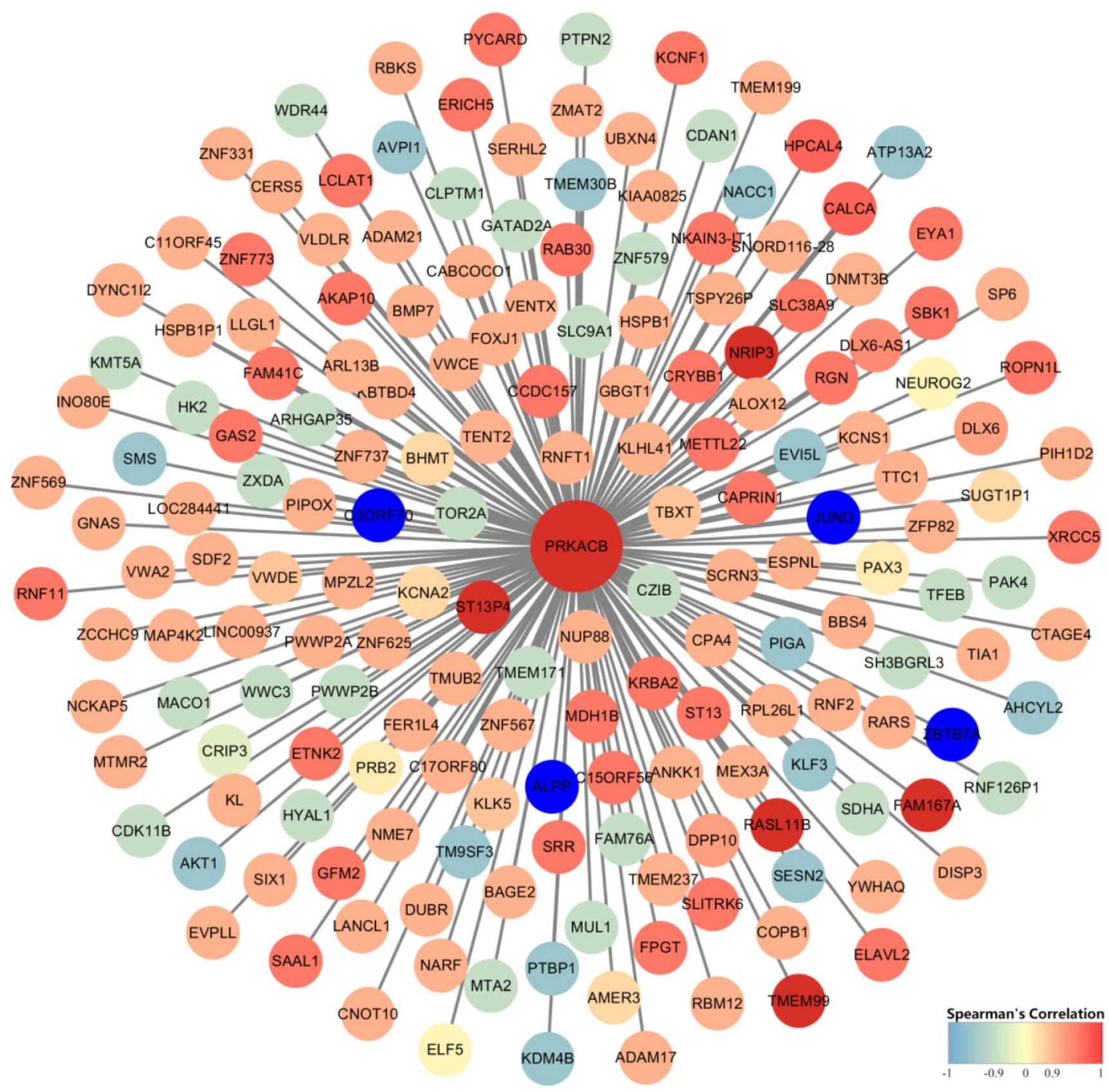

Figure 7. Construction of gene co-expression networks. Blue represents genes that are negatively related to PRKACB, and red points out genes that are positively related to PRKACB. The darker the color, the stronger the correlation.

\section{Acknowledgments}

This work was supported by grants from the Science and Technology Planning Project of Guangdong Province, China (No. 2017A030223006, 2016A020215128), the Science and Technology Planning Project of Guangzhou, China (No. 201704020077), the Research Fund of Guangdong General Hospital (No. y012015338), the Yuexiu Science and Information Center of Guangzhou Scientific Foundation (No. 2012-GX-046), the Second Batch of Scientific Research Projects of Dengfeng Plan (NO. DFJH201913), the Research Fund of
CSCO-Roche Oncology (NO. Y-2019Roche-190) and the Research Fund of CSCO-Hansoh Oncology (NO. Y-HS2019/2-050).

\section{Competing Interests}

The authors have declared that no competing interest exists.

\section{References}

1. Bray F, Ferlay I, Soerjomataram I, et al. Global cancer statistics 2018: GLOBOCAN estimates of incidence and mortality worldwide for 36 cancers in 185 countries. CA Cancer J Clin. 2018; 68:394-424.

2. Zhang L, Cao F, Zhang GY, et al. Trends in and Predictions of Colorectal Cancer Incidence and Mortality in China From 1990 to 2025. Frontiers In Oncology. 2019; 9:1-9. 
3. Chen W, Zheng R, Baade PD, et al. Cancer statistics in China, 2015. CA Cancer J Clin. 2016; 66:115-132.

4. Meyerhardt JA, Mayer RJ. Systemic therapy for colorectal cancer. N Engl J Med. 2005; 352:87-476.

5. Zhang F, Zhang Y, Zhao W, et al. Metabolomics for biomarker discovery in the diagnosis, prognosis, survival and recurrence of colorectal cancer: a systematic review. Oncotarget. 2017; 8:35460-35472.

6. Oh HH, Joo YE. Novel biomarkers for the diagnosis and prognosis of colorectal cancer. Intest Res. 2020; 18:168-183.

7. Zhu H, Lu W, Laurent C, et al. Genes encoding catalytic subunits of protein kinase A and risk of spina bifida. Birth Defects Res A Clin Mol Teratol. 2005; 73:591-596.

8. Skalhegg BS, Tasken K. Specificity in the cAMP/PKA signaling pathway. differential expression, regulation, and subcellular localization of subunits of PKA. Front Biosci. 1997; 2:d331-342.

9. Nakamura H, Arai $\mathrm{Y}$, Totoki $\mathrm{Y}$, et al. Genomic spectra of biliary tract cancer. Nat Genet. 2015; 47:1003-1010.

10. Singhi AD, Wood LD, Parks E, et al. Recurrent Rearrangements in PRKACA and PRKACB in Intraductal Oncocytic Papillary Neoplasms of the Pancreas and Bile Duct. Gastroenterology. 2019; 158:573-582

11. Simon EP, Freije CA, Farber BA, et al. Transcriptomic characterization of fibrolamellar hepatocellular carcinoma. Proc Natl Acad Sci U S A. 2015; 112:E5916-E5925.

12. Espiard S, Knape MJ, Bathon K, et al. Activating PRKACB somatic mutation in cortisol-producing adenomas. JCI Insight. 2018; 3:1-11.

13. Loilome W, Juntana S, Namwat N, et al. PRKAR1A is overexpressed and represents a possible therapeutic target in human cholangiocarcinoma. Int J Cancer. 2011; 129:34-44.

14. Zwart W, Griekspoor A, Berno V, et al. PKA-induced resistance to tamoxifen is associated with an altered orientation of ERalpha towards co-activator SRC-1. Embo j. 2007; 26:3534-3544

15. Wu KJ, Mattioli M, Morse HC, 3rd, et al. c-MYC activates protein kinase A (PKA) by direct transcriptional activation of the PKA catalytic subunit beta (PKA-Cbeta) gene. Oncogene. 2002; 21:7872-7882.

16. Padmanabhan A, Li X, Bieberich CJ. Protein kinase A regulates MYC protein through transcriptional and post-translational mechanisms in a catalytic subunit isoform-specific manner. J Biol Chem. 2013; 288:14158-14169.

17. Chen Y, Gao Y, Tian Y, et al. PRKACB is downregulated in non-small cell lung cancer and exogenous PRKACB inhibits proliferation and invasion of LTEP-A2 cells. Oncol Lett. 2013; 5:1803-1808.

18. Wang Z, Jensen MA, Zenklusen JC. A Practical Guide to The Cancer Genome Atlas (TCGA). Methods Mol Biol. 2016; 1418:111-141.

19. Gautier L, Cope L, Bolstad BM, et al. affy--analysis of Affymetrix GeneChip data at the probe level. Bioinformatics. 2004; 20:307-315.

20. Ritchie ME, Phipson $\mathrm{B}, \mathrm{Wu} \mathrm{D}$, et al. limma powers differential expression analyses for RNA-sequencing and microarray studies. Nucleic Acids Res. 2015; 43:e47-e47.

21. Robinson MD, McCarthy DJ, Smyth GK. edgeR: a Bioconductor package for differential expression analysis of digital gene expression data. Bioinformatics. 2010; 26:139-140

22. Nikolayeva O, Robinson MD. edgeR for differential RNA-seq and ChIP-seq analysis: an application to stem cell biology. Methods Mol Biol. 2014; 1150:45-79.

23. Gao J, Aksoy BA, Dogrusoz U, et al. Integrative analysis of complex cancer genomics and clinical profiles using the cBioPortal. Sci Signal. 2013; 6:pl1-pl1.

24. Cerami E, Gao J, Dogrusoz U, et al. The cBio cancer genomics portal: an open platform for exploring multidimensional cancer genomics data. Cancer Discov. 2012; 2:401-404.

25. Huang da W, Sherman BT, Lempicki RA. Systematic and integrative analysis of large gene lists using DAVID bioinformatics resources. Nat Protoc. 2009; 4:44-57.

26. Szklarczyk D, Morris $\mathrm{JH}$, Cook $\mathrm{H}$, et al. The STRING database in 2017: quality-controlled protein-protein association networks, made broadly accessible. Nucleic Acids Res. 2017; 45:D362-D368.

27. Killcoyne S, Carter GW, Smith J, et al. Cytoscape: a community-based framework for network modeling. Methods Mol Biol. 2009: 563:219-239.

28. Makondi PT, Chu CM, Wei PL, et al. Prediction of novel target genes and pathways involved in irinotecan-resistant colorectal cancer. PLoS One. 2017; 12:1-18.

29. Feng $Y Y$, Zeng DZ, Tong $Y N$, et al. Alteration of microRNA-4474/4717 expression and CREB-binding protein in human colorectal cancer tissues infected with Fusobacterium nucleatum. PLoS One. 2019; 14:1-13.

30. Wu S, Wu F, Jiang Z. Identification of hub genes, key miRNAs and potential molecular mechanisms of colorectal cancer. Oncol Rep. 2017; 38:2043-2050.

31. He H, Dai J, Zhuo R, et al. Study on the mechanism behind IncRNA MEG3 affecting clear cell renal cell carcinoma by regulating miR-7/RASL11B signaling. J Cell Physiol. 2018; 233:9503-9515

32. Dong-zhi C. Overexpression of NRIP3 gene inhibited metastasis in lymph node negative breast cancer. Journal of Shandong university (health science). 2019; 50:83-85.

33. Mao A, Chen $M$, Qin $Q$, et al. ZBTB7A promotes migration, invasion and metastasis of human breast cancer cells through NF-kappaB-induced epithelial-mesenchymal transition in vitro and in vivo. J Biochem. 2019; 166:485-493.
34. Liu S, Mao Q, Xue W, et al. High expression of ALPPL2 is associated with poor prognosis in gastric cancer. Hum Pathol. 2019; 86:49-56.

35. Elliott B, Millena AC, Matyunina L, et al. Essential role of JunD in cell proliferation is mediated via MYC signaling in prostate cancer cells. Cancer Lett. 2019; 448:155-167.

36. Irwandi RA, Khonsuphap P, Limlawan P, et al. miR-302a-3p regulates RANKL expression in human mandibular osteoblast-like cells. J Cell Biochem. 2018; 119:4372-4381.

37. Wang L, Liu J, Wang Q, et al. MicroRNA-200a-3p Mediates Neuroprotection in Alzheimer-Related Deficits and Attenuates Amyloid-Beta Overproduction and Tau Hyperphosphorylation via Coregulating BACE1 and PRKACB. Front Pharmacol. 2019; 10:1-12.

38. Wang J, Liu X, Wu H, et al. CREB up-regulates long non-coding RNA, HULC expression through interaction with microRNA-372 in liver cancer. Nucleic Acids Res. 2010; 38:5366-5383.

39. Nesterova MV, Johnson NR, Stewart T, et al. CpG immunomer DNA enhances antisense protein kinase A RIalpha inhibition of multidrug-resistant colon carcinoma growth in nude mice: molecular basis for combinatorial therapy. Clin Cancer Res. 2005; 11:5950-5955.

40. Sjoberg TJ, Kornev AP, Taylor SS. Dissecting the cAMP-inducible allosteric switch in protein kinase A RIalpha. Protein Sci. 2010; 19:1213-1221.

41. Manning G, Whyte DB, Martinez R, et al. The protein kinase complement of the human genome. Science. 2002; 298:1912-1934.

42. Michalides R, Griekspoor A, Balkenende A, et al. Tamoxifen resistance by a conformational arrest of the estrogen receptor alpha after PKA activation in breast cancer. Cancer Cell. 2004; 5:597-605. 